\title{
Article \\ Insights into the Role of the Microbiota and of Short-Chain Fatty Acids in Rubinstein-Taybi Syndrome
}

\author{
Elisabetta Di Fede ${ }^{1,+} \mathbb{D}$, Emerenziana Ottaviano ${ }^{1,+}$, Paolo Grazioli ${ }^{1}$, Camilla Ceccarani ${ }^{1,2} \mathbb{D}$, Antonio Galeone ${ }^{3}$, \\ Chiara Parodi ${ }^{1}$, Elisa Adele Colombo ${ }^{1}$ (D) Giulia Bassanini ${ }^{1}\left(\mathbb{D}\right.$, Grazia Fazio ${ }^{4}$, Marco Severgnini ${ }^{2}$, \\ Donatella Milani $^{5}\left(\mathbb{D}\right.$, Elvira Verduci ${ }^{1,6} \mathbb{D}$, Thomas Vaccari ${ }^{3} \mathbb{D}$, Valentina Massa ${ }^{1,7,} \neq \mathbb{D}$, Elisa Borghi ${ }^{1, \ddagger(\mathbb{D})}$ \\ and Cristina Gervasini $1,7, *, \ddagger(\mathbb{D})$
}

check for updates

Citation: Di Fede, E.; Ottaviano, E.; Grazioli, P.; Ceccarani, C.;

Galeone, A.; Parodi, C.; Colombo, E.A.;

Bassanini, G.; Fazio, G.; Severgnini, M.; et al. Insights into the Role of the Microbiota and of Short-Chain Fatty Acids in Rubinstein-Taybi Syndrome. Int. J. Mol. Sci. 2021, 22, 3621. https:// doi.org/10.3390/ijms22073621

Academic Editor: Rustam Aminov

Received: 22 February 2021

Accepted: 27 March 2021

Published: 31 March 2021

Publisher's Note: MDPI stays neutral with regard to jurisdictional claims in published maps and institutional affiliations.

Copyright: (c) 2021 by the authors. Licensee MDPI, Basel, Switzerland. This article is an open access article distributed under the terms and conditions of the Creative Commons Attribution (CC BY) license (https:/ / creativecommons.org/licenses/by/ $4.0 /)$.
1 Department of Health Sciences, Università degli Studi di Milano, 20142 Milan, Italy; elisabetta.difede@unimi.it (E.D.F.); emerenziana.ottaviano@unimi.it (E.O.); paolo.grazioli@unimi.it (P.G.); camilla.ceccarani@unimi.it (C.C.); chiara.parodi@unimi.it (C.P.); elisaadele.colombo@unimi.it (E.A.C.); giulia.bassanini@unimi.it (G.B.); elvira.verduci@unimi.it (E.V.); valentina.massa@unimi.it (V.M.); elisa.borghi@unimi.it (E.B.)

2 Institute of Biomedical Technologies, Italian National Research Council, Segrate, 20054 Milan, Italy; marco.severgnini@itb.cnr.it

3 Department of Biosciences, Università degli Studi di Milano, 20133 Milano, Italy; antonio.galeone@unimi.it (A.G.); thomas.vaccari@unimi.it (T.V.)

4 Tettamanti Research Center, Department of Pediatrics, Università degli Studi di Milano-Bicocca, MBBM Foundation/San Gerardo Hospital, 20900 Monza, Italy; grazia.fazio@unimib.it

5 Fondazione IRCCS Ca' Granda Ospedale Maggiore Policlinico, 20122 Milan, Italy; donatella.milani@policlinico.mi.it

6 Department of Pediatrics, Vittore Buzzi Children's Hospital, University of Milan, 20154 Milan, Italy

7 "Aldo Ravelli" Center for Neurotechnology and Experimental Brain Therapeutics, Università degli Studi di Milano, 20142 Milan, Italy

* Correspondence: cristina.gervasini@unimi.it; Tel.: +39-02-5032-3028

+ These authors contributed equally to this work.

$\ddagger$ These authors contributed equally to this work.

Abstract: The short-chain fatty acid butyrate, produced by the gut microbiota, acts as a potent histone deacetylase (HDAC) inhibitor. We assessed possible ameliorative effects of butyrate, relative to other HDAC inhibitors, in in vitro and in vivo models of Rubinstein-Taybi syndrome (RSTS), a severe neurodevelopmental disorder caused by variants in the genes encoding the histone acetyltransferases CBP and p300. In RSTS cell lines, butyrate led to the patient-specific rescue of acetylation defects at subtoxic concentrations. Remarkably, we observed that the commensal gut microbiota composition in a cohort of RSTS patients is significantly depleted in butyrate-producing bacteria compared to healthy siblings. We demonstrate that the effects of butyrate and the differences in microbiota composition are conserved in a Drosophila melanogaster mutant for CBP, enabling future dissection of the gut-host interactions in an in vivo RSTS model. This study sheds light on microbiota composition in a chromatinopathy, paving the way for novel therapeutic interventions.

Keywords: Rubinstein-Taybi syndrome; butyrate; microbiota; HDACi; histones

\section{Introduction}

Gene expression regulation is mediated by tightly balanced epigenetic mechanisms involving histone modifications, such as acetylation and methylation. Correct histone acetylation levels on lysine residues are fundamental for several physiological processes, including embryonic development [1,2]. Two classes of functionally antagonistic enzymes, the acetyltransferases (HAT) and deacetylases (HDAC), are known to modulate histone acetylation levels [3]. Histones hypoacetylation has been associated with alterations in synaptic plasticity, neuronal survival/regeneration, memory formation [4], while defects 
in epigenetic components acting on acetylation status cause several neurodevelopmen$\mathrm{tal} /$ malformation syndromes [5]. Among them, Rubinstein-Taybi syndrome (RSTS, OMIM $\# 180849, \# 613684)$ is a rare $(1: 125,000)$ autosomal-dominant disease characterized by a wide and heterogeneous spectrum of clinical signs [6]. These include intellectual disability of variable entity (ranging from mild to severe), postnatal growth deficiency, distinctive dysmorphisms, skeletal abnormalities (such as typical broad thumbs and large toes), multiple congenital anomalies (e.g., heart defects), and several additional clinical problems such as constipation [7]. Albeit the growth in height is constantly reduced in RSTS patients, growth in weight is reduced neonatally and in the first infancy, but at puberty an excessive weight gain is observed [8].

Most RSTS cases are caused by de novo monoallelic variants of one of two highly conserved genes: CREBBP, located at 16p13.3, coding for the CREB (cAMP response element-binding protein) binding protein (CBP) and EP300, mapping at 22q13.2, coding for the E1A-associated protein $\mathrm{p} 300$. CREBBP is found mutated in $>50 \%$ RSTS patients, while EP300 gene mutations have been described in a minor fraction of patients [9].

Somatic mutations in CREBBP and EP300 are reported in different benign and malignant tumors, and an association between RSTS patients and tumor development has been investigated. This disorder is related to an increased risk of malignancies up to $5 \%$, in particular involving cutaneous, hematological, and brain tumors such as pilomatrixoma, leukemia, and meningioma, respectively $[10,11]$.

CBP and p300 have ubiquitously expressed paralog proteins belonging to the lysine acetyl transferases (HAT) family [12]. CBP and p300 act as co-factors for transcription and are required in multiple pathways controlling cell growth, DNA repair, cell differentiation, and tumor suppression [13-16]. Their acetylation of target histone tails enables the opening of chromatin, thus promoting gene expression $[13,15,17]$.

In recent years, a novel class of compounds, termed HDAC inhibitors (HDACi), has been used to increase histone acetylation in different pathologies [18,19]. Preliminary studies testing the efficiency of HDACi to revert acetylation defects in RSTS lymphoblastoid cell lines (LCLs) supported the hypothesis that RSTS is caused by an acetylation imbalance [20]. Animal model studies introduced the idea that the chromatin alterations observed in RSTS could be reverted [21].

It has been demonstrated that protein acetylation can be modulated by the commensal microbial community (microbiota from here on) [22]. In fact, short-chain fatty acids (SCFAs), such as acetate, propionate, and butyrate, the most abundant products of anaerobic fermentation of the gut microbiota, can act as HDACi. Among SCFAs, butyrate is exclusively produced by commensal microorganisms and widely reported for its epigenetic activity, making it the most potent HDACi among natural compounds [23,24]. However, the role of butyrate or the composition of the microbiota in RSTS have not been investigated. Altered gut microbiota could itself affect the endogenous levels of SCFAs in patients, it could participate in their typical RSTS growth trend, characterized by a deficit in infancy and excessive weight gain after puberty, and/or it could contribute to the comorbidities often associated with RSTS, such as gastrointestinal discomfort [8].

On these premises, in the present study, we compared butyrate to other HDACi molecules in vitro on lymphoblastoid cell lines (LCLs) derived from RSTS patients. We have found it effective in modulating the acetylation impairment associated with reported CBP/p300 defects [20]. Remarkably, we also find that the microbiota of RSTS patients is poor in SCFA-producing bacteria, perhaps further contributing to acetylation imbalance. Finally, using Drosophila melanogaster, we model the effects of butyrate and microbiota alterations for future in vivo studies. Our work points to the importance of the microbiome in the pathogenesis and treatment of ultra-rare diseases. 


\section{Results}

\subsection{HDACi Exposure Counteracts Acetylation Imbalance in RSTS Lymphoblastoid Cell Lines (LCLs)}

To investigate the effect of SCFAs as HDACi in RSTS, we exposed LCLs derived from eight patients, four with CREBBP and four with EP300 mutations (Table S1) and seven healthy donors (HD) to sodium butyrate $(\mathrm{NaB})$, and we compared the effect to that of three other HDACi: trichostatin A (TSA), suberoylanilide hydroxamic acid (SAHA), and valproic acid (VPA) (Table S2). By AlphaLISA ${ }^{\circledR}$ assay, we analyzed the acetylation levels of lysine 27 of histone $\mathrm{H} 3$ (H3K27ac) in LCLs upon three different conditions: HDACi treatments, exposure to the vehicle (DMSO or $\mathrm{H}_{2} \mathrm{O}$ ), and untreated cells (Figure 1).

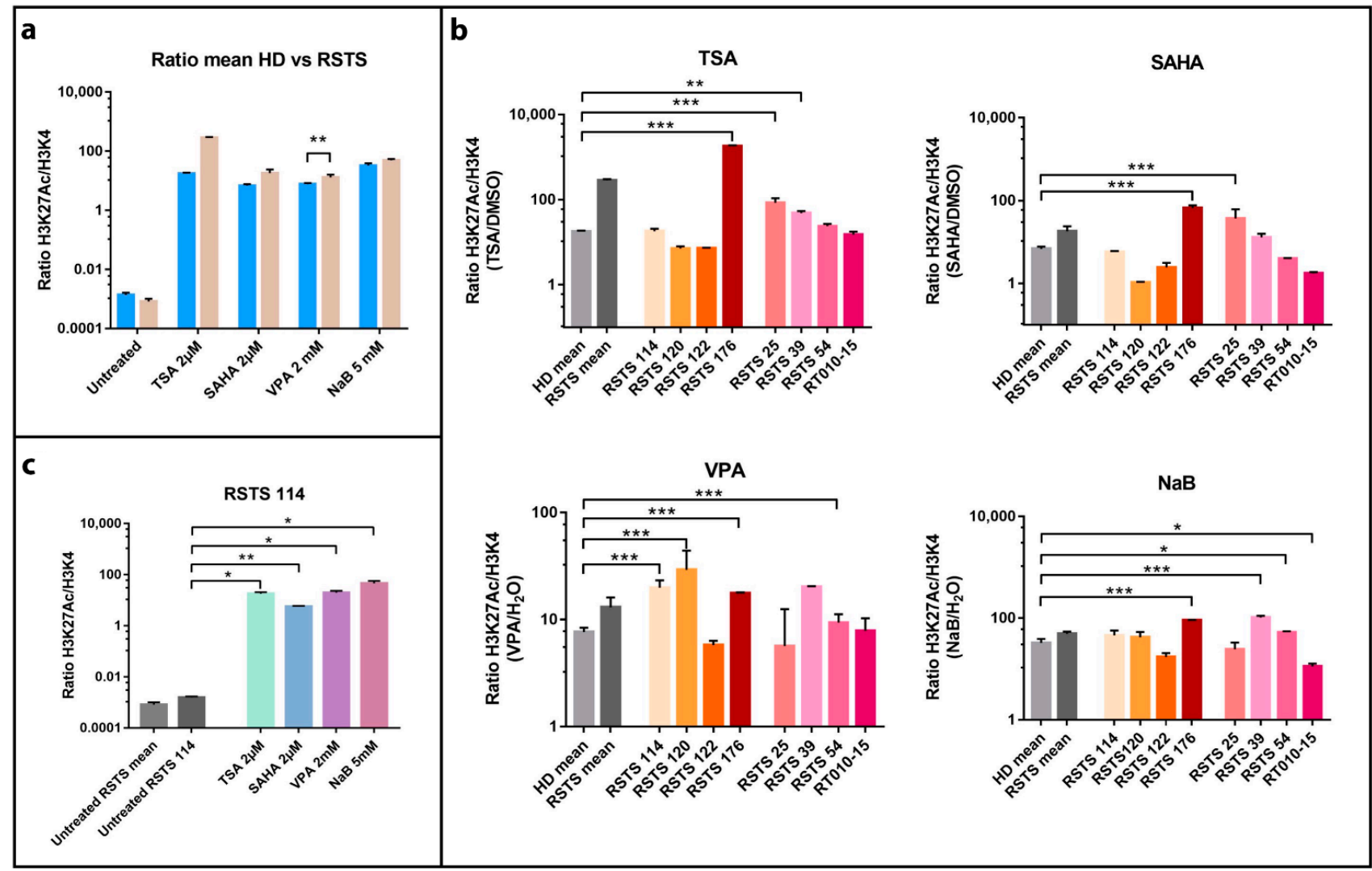

Figure 1. Histone acetylation on Rubinstein-Taybi syndrome (RSTS) lymphoblastoid cell lines (LCLs) upon acetyltransferases (HAT) and deacetylases (HDAC) inhibitors exposure. H3K27 acetylation levels normalized on H3K4 unmodified, assessed by AlphaLISA ${ }^{\circledR}$; levels of acetylation upon HDAC inhibitors (HDACi) are expressed as a ratio between the treatment and respective vehicle (HDACi/vehicle); on the Log scale, $Y$-axis H3K27 acetylation levels normalized, on X-axis lists of epigenetic treatments or untreated/treated single LCL or LCLs means. (a) Means of values of H3K27 acetylation in healthy donors (HD, in blue) and patients LCLs (RSTS, in pale brown) untreated and exposed to four different HDACi (trichostatin A (TSA) $2 \mu \mathrm{M}$, suberoylanilide hydroxamic acid (SAHA) $2 \mu \mathrm{M}$, valproic acid (VPA) $2 \mathrm{mM}$, and sodium butyrate (NaB) $5 \mathrm{mM}$ ). (b) H3K27 acetylation in eight RSTS LCLs (CREBBP LCLs in shades of red, EP300 LCLs in shades of pink) after exposure with the four different HDACi, compared to treated HD and RSTS means. (c) Insight on the single-patient response (RSTS 114) to the four compounds compared to untreated RSTS means and RSTS 114. Groups were compared using Student's $t$-test as statistical method $\left({ }^{*} p<0.05 ;{ }^{* *} p<0.01 ;{ }^{* * *} p<0.001\right)$. 
All the compounds succeeded in boosting histone acetylation in RSTS LCLs compared to healthy donor (HD) LCLs, with VPA exposure resulting highly significant $(p<0.01)$. This increment was particularly manifest in patient derived LCLs compared to untreated samples (Figure 1a).

We also observed that HDACi compounds induced a variable acetylation response, in a patient-specific manner when compared to treated HD LCLs (Figure 1b). As shown in Figure $1 \mathrm{~b}$, treatment with TSA $2 \mu \mathrm{M}$ boosted acetylation levels significantly in LCLs RSTS $176(p<0.001)$, RSTS $25(p<0.001)$ and RSTS $39(p<0.01)$, while SAHA $2 \mu \mathrm{M}$ showed highly significant effect on RSTS 176 and RSTS $25(p<0.001)$. VPA $2 \mathrm{mM}$ treatment particularly increased H3K27ac of RSTS 114, RSTS 120, RSTS 176, and RSTS $54(p<0.001)$, while exposure to $\mathrm{NaB} 5 \mathrm{mM}$ significantly affected acetylation of RSTS 176 and RSTS 39 $(p<0.001)$, RSTS 54, and RT010-15 ( $p<0.05)$.

Of note, when analyzing specific RSTS patient-derived LCLs response to HDACi compared to the relative untreated conditions, we observed that at least one HDACi significantly boosted acetylation and that RSTS-LCLs response varied among different drug treatments (Figure $1 \mathrm{c}$ and Figure S1). These data indicate that SCFAs as NaB show patient-specific acetylation increases, as is the case of other HDACi.

Considering HDACi applications as anticancer drugs for their role in cell cycle arrest, cell death, and immune-mediated mechanisms [25], we studied NaB and other HDACi effects on cell proliferation and apoptosis, performing Ki67 and Tunel assays upon exposure of LCLs with HDACi (Figures S2 and S3). For both assays, we did not observe a significant correlation with H3K27 acetylation (Figure S4), indicating that the HDACi rescue did not impair cell cycle progression or promote cell death under the experimental conditions used.

\subsection{RSTS Patients Are Depleted in the Major Butyrate-Producer Faecalibacterium spp.}

Because in vitro evaluation indicated that SCFAs can act as HDACi in RSTS, we focused on investigating the production of SCFAs by the commensal microbiota of the patients. To this end, we enrolled 23 RSTS subjects (mean age $10.2 \pm 6.4$ years; 12 females) and 16 healthy siblings (healthy donors, $\mathrm{HD}$ ), mean age $12.7 \pm 7.2$ years; 6 females), as a control group to minimize environmental factors having a well-recognized role on gut microbiota. The dietary survey revealed no differences in intake of macronutrients. However, energy intake was lower in RSTS subjects when compared to HD controls $(p=0.0054)$. Nutritional parameters are detailed in the relative supplementary table (Table S3) [26].

Microbiota profiling was performed through 16S rRNA gene-targeted sequencing. After quality filtering processes, we obtained a mean count of 90,759 reads per sample. The alpha-diversity analysis of the gut microbiota showed no significant differences between RSTS and HD fecal samples in terms of richness (Observed species: $p=0.255$; Phylogenetic Diversity (PD) whole tree: $p=0.279$-see Figure 2a) and of richness and evenness (Chao1: $p=0.151$; Shannon: $p=0.287$-see Figure S5). Beta-diversity analysis, instead, showed that RSTS fecal microbiota differed significantly from that of HD according to both unweighted $(p=0.013)$ and weighted $(p=0.022)$ Unifrac distances (beta-diversity, Figure $2 b)$. 

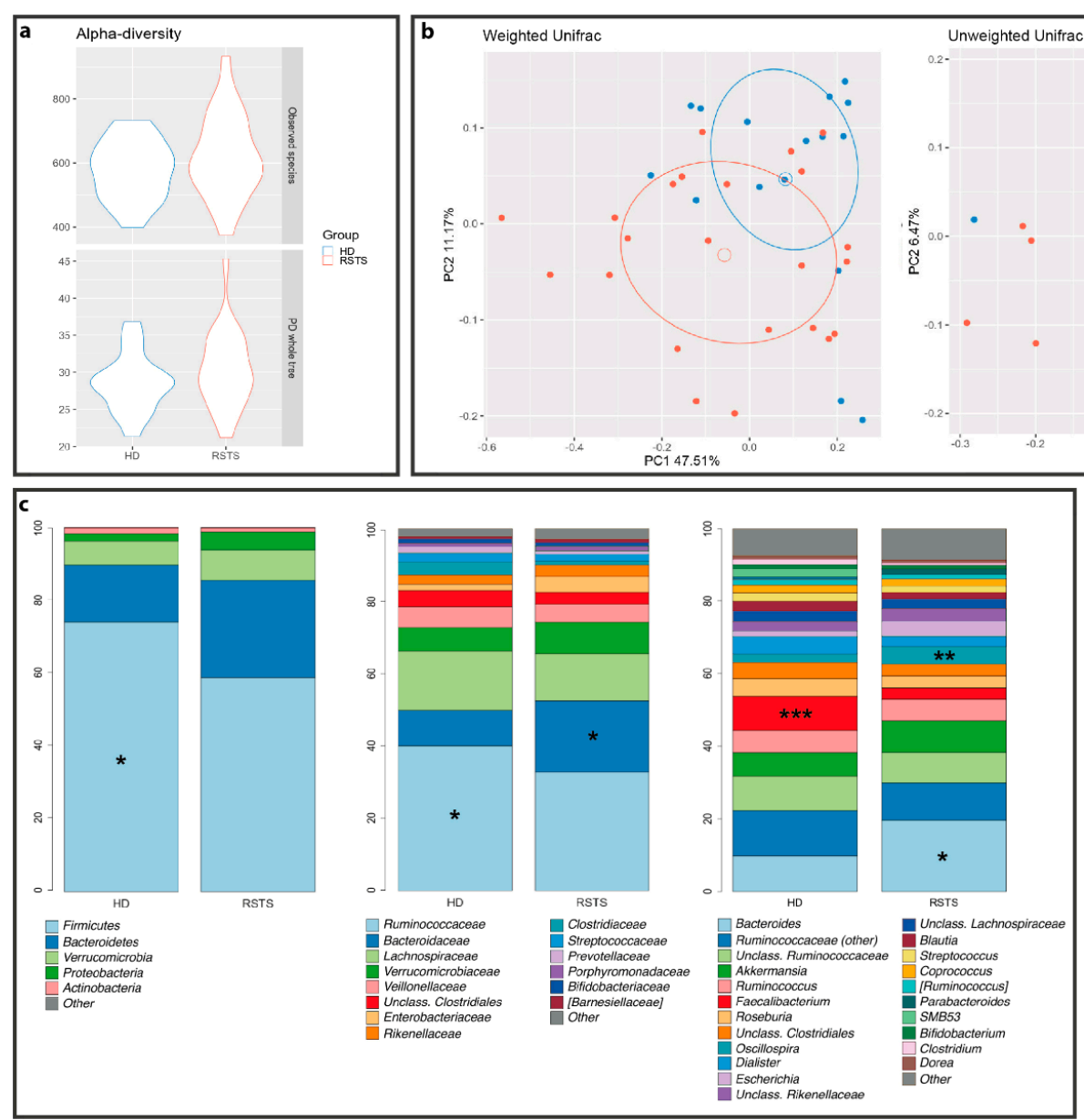
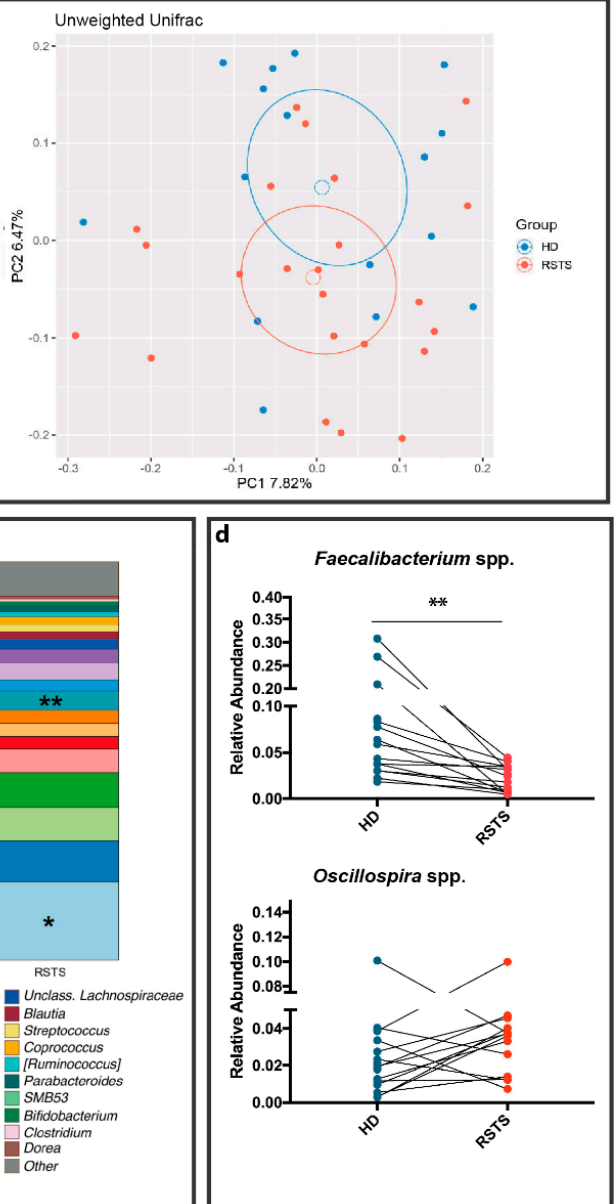

Figure 2. RSTS gut microbiota analysis. (a) alpha diversity. The violin plot shows biodiversity values for observed species and Faith's phylogenetic metrics. No statistically relevant differences were seen. (b) Principal coordinate analysis (PCoA) according to weighted and unweighted Unifrac distances. Microbial communities are statistically different (Adonis test: unweighted $p=0.019$; weighted $p=0.023$ ). The first and second principal coordinates are shown in the plot for both distances. (c) Bacterial composition of HD and RSTS groups. Relative taxonomic abundances are shown at phylum, family, and genus phylogenetic levels. All bacterial taxa present at $<1 \%$ relative abundance were grouped into the "Other" classification. ${ }^{* *}: p<0.005 ;{ }^{* *}: p<0.01 ;{ }^{*}: p<0.05$. (d) Faecalibacterium spp. and Oscillospira spp. relative abundances (both significantly different between RSTS and HD) were compared within matched family members (patient/sibling, $n=16$ ). For Oscillospira we did not observe a common pattern; Faecalibacterium spp. was significantly reduced in RSTS ( $p=0.0021$, Wilcoxon signed-rank test).

The overall composition of the intestinal microbiota (Figure 2c; Table S4) showed a decreased relative abundance of the Firmicutes phylum (58.5\% in RSTS vs. $73.4 \%$ in HD, $p=0.019)$, of the Ruminococcaceae family $(32.2 \%$ vs. $41.9 \%, p=0.049)$ and of the SCFAproducing Faecalibacterium spp. ( $3.3 \%$ vs. $9.8 \%$ in HD, $p=0.001)$ in RSTS subjects. On the other hand, RSTS samples showed an enrichment in the Bacteroidaceae family and in Bacteroides spp. (21.1\% vs. $10.3 \%, p=0.021)$, as well as in Oscillospira spp. (5.1\% vs. $2.4 \%$ in HD, $p=0.007$ ). Matched-pair analysis (Wilcoxon signed-rank test), performed on RSTS/sibling pairs showed a significant and environment-independent decrease $(p=0.0021)$ in Faecalibacterium spp. (Figure 2d).

Afterward, we directly measured SCFA abundance in patient fecal samples. A decreasing trend in butyrate content was observed $(4.13 \pm 1.40 \mathrm{vs.} 5.14 \pm 1.79 \mathrm{mg} / \mathrm{g}$ feces, $p=0.0741$, Mann-Whitney test), whereas acetate, propionate, and branched-chain fatty acids (iso-butyrate and iso-valerate) concentrations were similar $(p=0.194, p=0.874$, $p=0.786$, and $p=0.467$, respectively). These data indicate that RSTS patient microbiota produces low levels of endogenous butyrate. 


\subsection{HDACi Exposure Leads to Partial Rescue of RSTS Phenotype in Drosophila CBP Mutants}

To establish an in vivo model to study the effect of SCFAs and the microbiome on RSTS pathogenesis, we analyzed Drosophila melanogaster mutants in the CBP homolog nejire (nej). Homozygous nej loss of function results in abnormal embryonic development leading to early lethality $[27,28]$. In particular, mutations in nej lead to defects in morphology caused by misregulation of wingless $(\mathrm{wg})$ and other signaling pathways at stage 9 of embryonic development [27]. To test whether $\mathrm{NaB}$, or VPA as a control HDACi, upon supplementation in the food could modulate emergence of nej mutant defects in vivo, we collected embryos deposed by females fed with the drugs and assessed their morphology at stages 8-12 (Figure 3 and Figure S6).

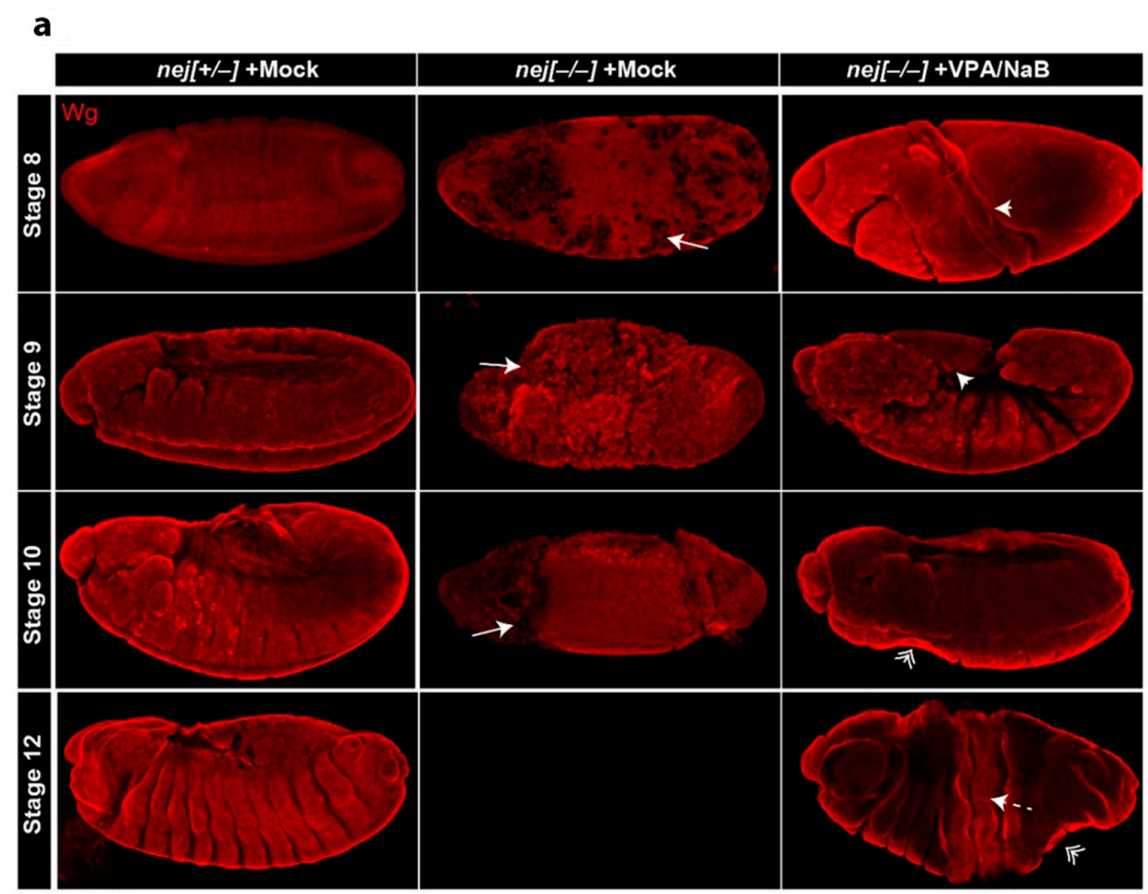

b

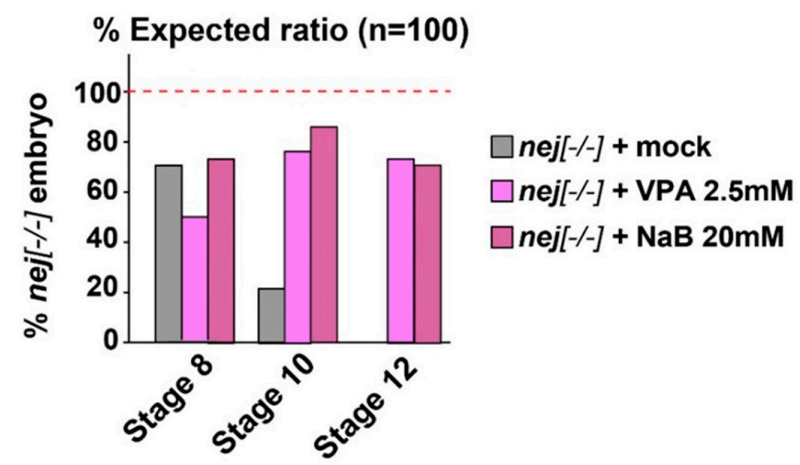

Figure 3. Developmental and morphological defects in nej mutant embryos are partially ameliorated by VPA or NaB treatment. (a) wingless (wg) staining of nej mutant embryos treated as indicated. Representative projections of confocal imaging from stages 8 to 12 are shown. The defects detected in nej mutant embryos with or without treatment include loss or uneven wg staining (Drilled, arrow), twisting (arrowhead), presence of bottlenecks (double-arrowheads), or cracks (dashed-arrow). (b) Quantification of embryo survival and of the above phenotypes in nej mutant embryos at stages 8 to 12. VPA or NaB treatments partially rescue embryo development, allowing nej mutant embryos to survive beyond stage 8 although with aberrant phenotypes, with significant embryo survival at stage 10 compared to untreated embryos $(p>0.01)$. Student's t-test was used as statistical method for comparing nej groups survival, with $p<0.05$ considered significant. 
Compared to $n e j^{+/-}$siblings, we have found that the majority of $n e j^{-/-}$embryos die between stages 8 and 10 with the reported twisting of embryo morphology [28] (Figure 3a, quantification in Figure $3 b$ ). Upon NaB or VPA treatment, $n e j^{-/-}$embryos display a partially rescued embryonic development, statistically significant at stage $10(p<0.01)$. Importantly, both treatments extended the survival of $n e j^{-/-}$embryos to stage 12, although twisting of the embryos is morphologically visible as bottleneck and cracks (Figure 3 and Figure S6). The reported twisting phenotype in treated $n e j^{-/}$embryos is often less dramatic than in untreated $n j^{-/-}$embryos allowing segmentation. Notably, both VPA and $\mathrm{NaB}$ treatment did not show any developmental delay or morphological defects in control embryos (data not shown). Altogether, these results indicate that $\mathrm{NaB}$ acts as a HDACi in vivo to ameliorate the developmental defects associated with acute loss of CBP homologs.

\subsection{The Fly Gut Microbiota of Heterozygous Drosophila CBP Mutants}

Because RSTS patients possess a defective microbiota, we analyzed that of heterozygous $n e j\left(n e j^{+/-}\right)$flies. In contrast to $n e j^{-/-}$embryos, $n e j^{+/-}$animals progress to adulthood and display no overt defects. However, they have been shown to reveal genetic interaction with genes involved in developmental processes regulated by CBP [28]. Hence, we reasoned that adult $n e j^{+/-}$animals could be used to investigate the fly gut microbiota.

A total of 10 samples were obtained from the two experimental groups ( $y w$ and $n e j^{+/-}$), with five replicates each (three dissected guts in each replicate, with a total of 15 flies per group).

Alpha diversity metrics (Figure 4a) revealed that nej gut microbiota were enriched in low abundant species (Chao1 metric, $p=0.005$ ), whereas the phylogenetic diversity between groups showed no significant differences (PD whole tree metric, $p=0.668$ ).

Similarly, beta-diversity analysis (Figure 4 b) highlighted clear discrimination between $y w$ and nej microbial communities considering both abundant and rare species within the microbiota (unweighted Unifrac distance, $p=0.007$ ),

As for the bacterial composition, the Firmicutes phylum was found more abundant in the $n e j$ flies $(64.7 \%$ vs. $53.9 \%$ in $y w)$, with a concurrent reduction of Proteobacteria (31.6 vs. $41.1 \%$ in $y w)$. The taxonomic analysis revealed a remarkable increase in Lactobacillaceae (58.8\% in nej vs. $7.5 \%$ in $y w ; p=0.0000254)$, and a profound decrease in the Enterococcaceae family $(0.2 \%$ vs. $12.9 \%$ in $y w ; p=0.00784)$ (Figure 4 c). These results were confirmed at genus level, with a significant enrichment in Lactobacillus $(58.4 \%$ in nej vs. $3.4 \%$ in $y w ; p=0.00000806)$ and a depletion in Enterococcus ( $0 \%$ vs. $3 \%$ in $y w ; p=0.00772)$ (data not shown). 

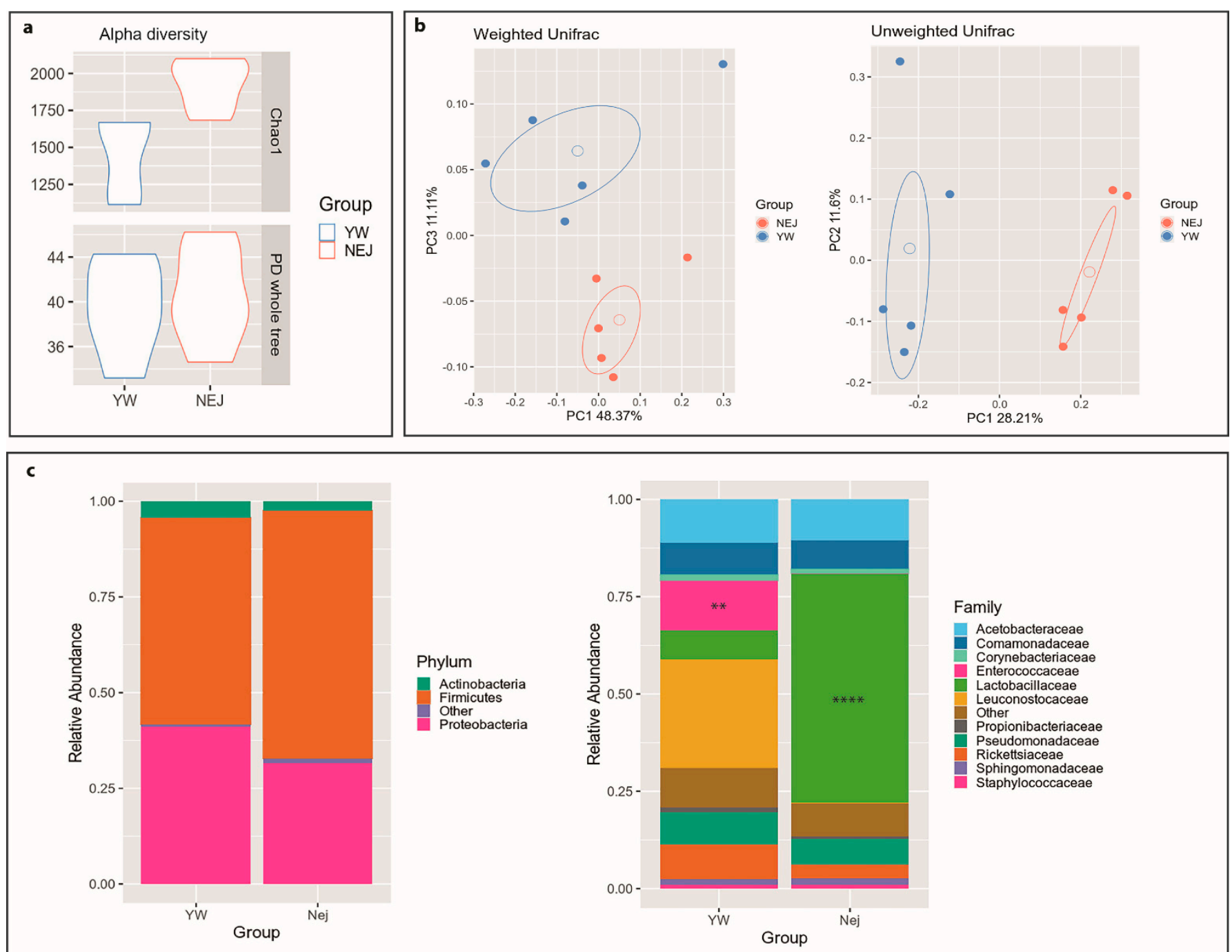

Figure 4. Drosophila gut microbiota analysis. Gut microbial communities were characterized by $16 \mathrm{~S}$ rRNA gene sequencing. (a) Alpha diversity. Violin plots show biodiversity values for Chao1 $(p=0.005)$ and Faith's phylogenetic metrics (PD whole tree; $p=0.668)$. (b) Principal coordinate analysis (PCoA) for both Unifrac distances. (Adonis test: weighted $p=0.216$; unweighted $p=0.007)$. (c) Relative taxonomic abundances of the gut bacterial composition in yw and nej flies at phylum and family levels. All bacterial taxa present at $<1 \%$ relative abundance were grouped into the "Other" classification. $* * * *: p<0.001 ; * *: p<0.01$.

\section{Discussion}

Current therapeutic approaches for RSTS patients are not targeted towards modulation of acetylation and are rather directed towards alleviating clinical symptoms and preventing possible known comorbidities. Common interventions include behavioral support and surgical procedures for the correction of orthopedic or cardiac malformations. In this context, exploring the effects of drugs with an established and specific molecular function in acetylation in preclinical studies is a fundamental step to devise effective future therapy. In the case of RSTS, HDACi are available and have already been used to treat neurological disorders [29]. Hence, the presented work explored the effects of HDAC inhibition in RSTS experimental models, focusing on natural SCFAs such as butyrate.

Treatment with a number of HDACi used in cancer therapy [30-33] showed a general boost in histone acetylation levels in RSTS patient-derived cell lines. Such increment was significant in a patient-specific manner. Each line derived from different RSTS patients with discrete pathogenic variants responded differently to tested compounds. These data provide evidence for HDACi's ability to restore acetylation levels in an in vitro model of 
RSTS, strongly pointing to the possibility of future therapies tailored to individual patients, a central tenet of personalized medicine.

HDACi are tested in oncology trials for their ability to stop tumor cell proliferation by inducing selected and dose-dependent apoptosis [34]. Thus, our observation that selected HDACi dosing does not modulate cell proliferation or death in RSTS cells indicates that HDACi can boost acetylation at a sub-toxic concentration, at least in vitro.

Due to the HDACi function exerted by SCFAs, they have been tested in clinical trials for recurrent malignant gliomas or myelodysplastic syndrome and they have also been studied in vitro against Burkitt lymphoma, primary acute myeloid leukemia, retinoblastoma, medulloblastoma, prostate cancer, and hepatocellular and colon carcinoma [35,36].

Importantly, NaB, a natural SCFA with potent $\mathrm{HDACi}$ activity [37], performed on par with other HDACi in RSTS cells. We propose that it should be evaluated for the treatment of patients, considering that it is present in human diets as a product of the human microbiota or a well-tolerated supplement.

To investigate whether butyrate is normally produced by commensal bacteria of RSTS patients, we sequenced the V3-V4 hypervariable regions of the 16S rRNA genes and measured SCFAs as main microbial metabolites. While we scored no differences in nutritional parameters and in microbiota biodiversity in patients versus healthy siblings, we observed a distinct and highly interesting microbial signature characterized by loss of the butyrate-producer genus Faecalibacterium [38] in the microbiota of RSTS patients. Such change could participate in the syndrome comorbidity insurgence, perhaps in the gut, and further study should now aim at elucidation of possible genetic-microbial additive negative effects. Interestingly, a reduction of Faecalibacterium relative abundance was also reported in patients affected by Rett syndrome, autism spectrum disorder, and down syndrome, suggesting a shared microbiota signature in neurodevelopmental disorders [39].

A recent study reported that a ketogenic diet, highly impacting microbiota composition and metabolism [40], induces the production of deacetylase inhibitors in a mouse model of Kabuki syndrome (OMIM\# 147920, \# 300867), a rare disease sharing traits and histone modification defect with Rubinstein-Taybi syndrome [41]. Thus, nutritional interventions could also aim at rebalancing the microbiota of RSTS patients. In line with this approach, brain functions and behavior appear more and more influenced, through a bottom-up modulation, by the gut microbiota [42].

It is worth noting that carbohydrates, and in particular fermentable dietary fibers, the most important substrates for short-chain fatty acid production [43], were very similar in RSTS patients and healthy siblings, inconsistent with the reduction in the relative abundance of Faecalibacterium genus, or with the lower butyrate fecal concentration. Nutritional recommendations for RSTS comorbidity management are currently lacking as no studies focused on this. Our findings represent a starting point for the evaluation of specific nutritional regimens, which could also shed light on the basis of observed differences.

Studies with nej flies underscored the role of CBP during embryogenesis and as coactivators of critical signaling pathways involved in patterning [28,44]. In support of results from our in vitro RSTS model, we observed that rearing flies with food supplemented with $\mathrm{NaB}$ lead to partial rescue of embryogenesis and patterning, suggesting that nutritional intervention may ameliorate RSTS traits in vivo.

However, even if the Drosophila model has been used for investigating how different levels of nutrients and drugs influence the development and the metabolic phenotypes of emerging Drosophila embryos [45], our data are model behavior of oviparous animals, in which development occurs outside the mother's body. In mammals, maternal HDACi cross the placenta and affect embryogenesis; hence any future intervention should be envisaged after birth when the mammalian central nervous system is still developing.

Lack of an anoxic compartment in the Drosophila gut shapes a microaerophilic microbiota constituted, in laboratory strains, by few genera [46]. The most abundant taxa are Firmicutes, mainly Lactobacillus spp. and alpha-Proteobacteria, mainly Acetobacteraceae. Despite evolutionary divergence with the human microbiota, recent studies showed that an 
altered relative abundance of these genera can result in gut homeostasis disturbance [47], growth delay [48], and behavioral changes [49]. Considering the results obtained from microbiota analysis of RSTS patients, we analyzed the microbial community of heterozygous nej insects compared to control animals. Results showed that, accounting for the species-specificity of the microbiota, the differences observed in RSTS patients compared to healthy siblings are recapitulated in RSTS flies compared to control animals, suggesting that patients-microbiota interactions could be modeled in Drosophila.

Overall, our results are in line with other studies [12,20,50], making a strong case for HDACi drug repurposing for future RSTS therapy. We envisage that the use of Drosophila melanogaster as an information rich in vivo RSTS model will speed up the transition from preclinical studies to clinical practice.

\section{Materials and Methods}

\subsection{Cell Cultures}

Lymphoblastoid cell lines (LCLs) from eight different RSTS patients (four carrying CREBBP mutations and four carrying EP300 mutations, listed in Table S1) [20,51-53] and seven healthy donors were obtained in collaboration with the Gaslini Genetic Bank service (Telethon Network of Genetic Biobanks); their use was approved by Ethics Committee of Università degli Studi di Milano (Comitato Etico number 99/20, 17 November 2020). Cells were maintained in RPMI 1640 culture medium supplemented with L-glutamine (Euroclone, Pero, Italy), 20\% fetal bovine serum (Euroclone, Pero, Italy), and penicillin/streptomycin (Euroclone, Pero, Italy), and cultured in an incubator with $5 \% \mathrm{CO}_{2}$ at $37^{\circ} \mathrm{C}$.

LCLs were exposed to four different HDAC inhibitors: Trichostatin A (TSA) (sc-3511, Santa Cruz Biotechnology, Dallas, TX, USA), Suberoylanilide hydroxamic acid (SAHA) (MK0683, Selleckchem, Houston, TX, USA), Valproic acid (VPA) (P4543, Sigma Aldrich, St. Louis, MO, USA), and Sodium Butyrate (NaB) (B5887, Sigma-Aldrich, St. Louis, MO, USA). We tested three different concentrations for each HDACi (Table S2) [54-59] and selected the maximum dose and timing of exposure, ensuring acceptable LCLs survival (data not shown). Cells were incubated with vehicles $\left(\mathrm{H}_{2} \mathrm{O}\right.$ or DMSO) at the maximum time (24 h), TSA $2 \mu \mathrm{M}$ for $2 \mathrm{~h}$, SAHA $2 \mu \mathrm{M}$ for $24 \mathrm{~h}$, VPA $2 \mathrm{mM}$ for $24 \mathrm{~h}$, or NaB $5 \mathrm{mM}$ for $24 \mathrm{~h}$ as suggested from the literature (Table S2). Data were normalized on untreated cells and in vehicles for accounting for proliferation rate differences in basal condition between HD and RSTS lines.

\subsection{AlphaLISA ${ }^{\circledR}$ Assay}

After treatments, lymphoblastoid cellular pellets were obtained by centrifugation and frozen at $-80^{\circ} \mathrm{C}$. An amount of 10,000 cells/well resuspended in $60 \mu \mathrm{L}$ of culture media was used in order to perform AlphaLISA ${ }^{\circledR}$ assay (PerkinElmer, Waltham, MA, USA) according to the manufacturer's protocol. Briefly, cells were incubated $15 \mathrm{~min}$ with Cell-Histone Lysis buffer and $10 \mathrm{~min}$ with Cell-Histone Extraction buffer; $30 \mu \mathrm{L}$ of lysates were incubated with $10 \mu \mathrm{L}$ of Acceptor mix $1 \mathrm{~h}$ at room temperature (RT) and then $10 \mu \mathrm{L}$ of Donor mix was added overnight at RT. Replicates were tested with both AlphaLISA Acetylated-Histone H3 Lysine 27 (H3K27ac) Cellular Detection Kit (AL720, PerkinElmer, Waltham, MA, USA) and AlphaLISA unmodified Histone H3 Lysine 4 (H3K4) Cellular Detection Kit (AL719, PerkinElmer, Waltham, MA, USA) for normalization. PerkinElmer EnSight ${ }^{\mathrm{TM}}$ plate reader was used for the detection of the chemiluminescent signal.

\subsection{Ki67 and TUNEL Assay}

After treatments, at least $1.5 \times 10^{4}$ LCLs were seeded in duplicate on SuperFrost Plus slides (Thermofisher Scientific, Waltham, MA, USA) through 5 min of cytospin at $500 \mathrm{rpm}$, followed by $10 \mathrm{~min}$ of incubation with PFA $4 \%$ and washed. Slides were stored at $4{ }^{\circ} \mathrm{C}$ until Ki67 or TUNEL assays were performed. 
Briefly, for Ki67 assay slides, samples were put in a wet chamber and cells permeabilized with PBT buffer (Phosphate-Buffered Saline (PBS) with $0.2 \%$ Triton) for $10 \mathrm{~min}$ at room temperature (RT); blocking of non-specific sites was obtained by slide incubation with PBT supplemented with $10 \%$ FBS for 30 min at RT. Slides were first incubated overnight at $4{ }^{\circ} \mathrm{C}$ with the anti-Ki67 antibody (\#9129 Cell Signaling, Danvers, MA, USA, 1:400), washed with PBT, and then incubated with Alexa-488 anti-Rabbit secondary antibody (\#6441-30 SouthernBiotech, Birmingham, AL, USA, 1:250) for $2 \mathrm{~h}$. Slides were washed with PBT and water, mounted with EverBrite Mounting Medium with DAPI (23002, Biotium, Landing Parkway Fremont, CA, USA), and fluorescent microscopic images of proliferative cells (Ki67+) were acquired and analyzed with ImageJ software (National Institute of Health, Bethesda, MD, USA).

Terminal deoxynucleotidyl transferase (TdT) dUTP Nick-End Labeling (TUNEL) assay was performed using In Situ Cell Death Detection kit, AP (Roche Diagnostics, Basilea, Switzerland), in order to detect apoptotic cells, according to manufacturer's protocol. Cells, previously seeded on slides were incubated with a permeabilization solution $(0.1 \%$ Triton $100 \mathrm{X}$ and $0.1 \%$ sodium citrate) for $2 \mathrm{~min}$ at $4{ }^{\circ} \mathrm{C}$, then washed with PBS and incubated with TUNEL mixture (composed by Enzyme Solution added to Label Solution) in a wet chamber for $1 \mathrm{~h}$ at $37^{\circ} \mathrm{C}$. After 3 PBS washes, slides were incubated with Converter AP for $30 \mathrm{~min}$ at $37^{\circ} \mathrm{C}$ and then with Substrate Solution ( $2 \%$ NBT/BCIP stock solution in NBT/BCIP Buffer) for $10 \mathrm{~min}$ at RT and dark. Finally, following PBS washes, mounted with DABCO mounting medium and brightfield microscopic images of apoptotic cells (TUNEL+) were acquired and analyzed with ImageJ software (National Institute of Health, Bethesda, MD, USA).

Both fluorescent and brightfield slide images were acquired by NanoZoomer S60 Digital Slide Scanner (Hamamatsu Photonics, Hamamatsu City, Japan) at $20 \times$ and $80 \times$ magnification, and two randomly selected fields for each experimental group at $20 \times$ were selected for blinded cells counts by three different operators. Panel images of Ki67+ cells were instead acquired by confocal microscopy A1/A1R (Nikon Corporation, Tokyo, Japan) at $60 \times$ and $100 \times$ magnification. The number of Ki67+ and TUNEL+ cells was normalized on the total cell number per image.

\subsection{Subject Recruitment and Sampling for Gut Microbiota Profiling}

For this study, 23 RSTS subjects and 16 healthy siblings were enrolled. All subjects were recruited in collaboration with the Italian family RSTS association "Associazione RTS Una Vita Speciale ONLUS".

For both patients and controls, exclusion criteria were treatments with antibiotic and/or probiotic/prebiotic assumption during the previous 3 months. For RSTS patients, inclusion criteria were confirmed clinical diagnosis with $(20 / 23)$ or without $(3 / 23)$ demonstrated CREBBP/EP300 mutation. RSTS diagnosis of all patients was confirmed by an expert geneticist $(\mathrm{DM})$ and genetic tests were performed in our laboratory $(\mathrm{CG})$.

In conjunction with the stool sample collection, a 3 day dietary survey (preceding the sample collection) was filled by caregivers. Dietary food records were processed using commercially available software (ePhood V2, Openzone, Bresso, Italy).

The study was approved by the Ethics Committee of San Paolo Hospital in Milan (Comitato Etico Milano Area 1, Protocol number 2019/EM/076, 2 May 2019); written informed consent was obtained from enrolled subjects or caregivers.

\subsection{Bacterial DNA Extraction and 16S rRNA Gene Sequencing of Human Gut Microbiota}

Bacterial genomic DNA in stool samples was extracted as previously described [60] by using the Spin stool DNA kit (Stratec Molecular, Berlin, Germany), according to the manufacturer's instructions. Briefly, after homogenizing fecal samples in the lysis buffer for inactivating DNases, Zirconia Beads II were added for a complete lysis of bacterial cells by using TissueLyser LT. Bacterial lysates were then mixed with InviAdsorb reagent, a step designed to remove PCR inhibitors. Bacterial DNA was eventually eluted in $100 \mu \mathrm{L}$ of buffer. Then, $25 \mathrm{ng}$ of extracted DNA was used to construct the sequencing library. The V3-V4 
hypervariable regions of the bacterial $16 \mathrm{~S}$ rRNA were amplified with a two-step barcoding approach according to the Illumina 16S Metagenomic Sequencing Library Preparation (Illumina, San Diego, CA, USA). Library quantification was determined using the DNA High Sensitivity Qubit kit (Thermofisher Scientific, Waltham, MA, USA) and Agilent 2100 Bioanalyzer System (Agilent, Santa Clara, CA, USA); libraries were pooled and sequenced on a MiSeq platform (Illumina, San Diego, CA, USA) in a $2 \times 250$ bp paired-end run. Obtained $16 \mathrm{~S}$ rRNA gene sequences were analyzed using PANDAseq [61], and low-quality reads were filtered and discarded. Reads were then processed using the Quantitative Insights Into Microbial Ecology (QIIME) pipeline (release 1.8.0) [62] and clustered into Operational Taxonomic Unit (OTUs) at $97 \%$ identity level and discarding singletons (i.e., OTUs supported by only 1 read across all samples) as likely chimeras. Taxonomic assignment was performed via the Ribosomal Database Project (RDP) classifier [63] against the Greengenes database (version 13_8; ftp://greengenes.microbio.me/greengenes_release/gg_13_8_otus, accessed on 22 February 2021), with a 0.5 identity threshold. Alpha-diversity was computed using the Chao1, the number of OTUs, Shannon diversity, and Faith's Phylogenetic Diversity whole tree (PD whole tree) metrics throughout the QIIME pipeline. Beta-diversity was assessed by weighted and unweighted UniFrac distances [64] and principal coordinates analysis (PCoA).

\subsection{Fecal Short-Chain Fatty Acid Quantification}

Concentrations of acetate, propionate, iso-butyrate, butyrate, and iso-valerate were assessed according to Bassanini et al. [65]. The measurement of SCFAs was performed by gas chromatography, using a Varian model 3400 CX Gas chromatograph fitted with FID detector, split/splitless injector, and a SPB-1 capillary column ( $30 \mathrm{~m} \times 0.32 \mathrm{~mm} \mathrm{ID,} 0.25 \mu \mathrm{m}$ film thickness; Supelco, Bellefonte, PA, USA). Calibration curves of SCFAs in concentration between 0.25 and $10 \mathrm{mM}$ were constructed to obtain SCFAs quantification, and $10 \mathrm{mM}$ 2-ethylbutyric acid was used as an internal standard. Results are expressed as mg/g of dry weight of feces.

\subsection{Drosophila Melanogaster Stocks and HDACi Feeding}

The following fly strains were used in this study: Drosophila yw strain used as control and $w\left[^{*}\right] P\{w[+m C]=l a c W\} n e j[P] / F M 7 c$ known as nejire (nejP/+) mutant strain (\#3728; Bloomington Drosophila Stock Center, Bloomington, IN, USA). nejP contains a P-element 347bp upstream of the second exon of nej gene and behaves as a loss of function mutant [27]. Flies were maintained and raised into vials containing a standard food medium composed of yeast, cornmeal, molasses, agar, propionic acid, tegosept, and water. All the strains were kept at $25^{\circ} \mathrm{C}$. To prepare food with HDACi, stock solutions of VPA ( 1 and $\left.2.5 \mathrm{mM}\right)$ and $\mathrm{NaB}(10$ and $20 \mathrm{mM})$ solutions were diluted 1:10 in the food before solidification but under $65{ }^{\circ} \mathrm{C}$ to prevent heat damage of the compounds.

\subsection{Fly Treatment and Embryo Immunostaining}

To identify homozygous animals in sibling crosses, nej mutants were balanced over FM7, kr-GAL4 UAS-GFP chromosome. Adult nej mutant flies were life cycle-synchronized and treated with mock, VPA $(2.5 \mathrm{mM})$ or $\mathrm{NaB}(20 \mathrm{mM})$ for four days. The drugs were mixed with standard food as described above at room temperature. For egg collection, adult flies were placed in cages for $4 \mathrm{~h}$ and then removed. Fertilized eggs were collected after $8 \mathrm{~h}$ and stained as previously described [66]. Briefly, embryos were collected, dechorionated and fixed with a mixture of $4 \%$ paraformaldehyde and heptane. Following washes, embryos were permeabilized and blocked with PBT (PBS containing 0.1\% Triton X-100 and 1\% BSA) for $3 \mathrm{~h}$. Staining was performed overnight with primary mouse anti-wg 1:50 (4D4, Developmental Studies Hybridoma Bank, Iowa City, IA, USA). Secondary goat anti-mouseCy3 (1:500) was used for $2 \mathrm{~h}$. Images were taken with a Nikon AR1 confocal microscope using a 10X objective (Nikon Corporation, Tokyo, Japan). 


\subsection{Bacterial DNA Extraction and $16 S$ rRNA Gene Sequencing of Drosophila Melanogaster Gut Microbiota}

For microbiota sequencing, adult fly guts were dissected to avoid environmental contamination. Briefly, flies were anesthetized in ice for $5 \mathrm{~min}$ in a Petri dish and transferred, one by one, to the dissection dish and immersed in $50 \mu \mathrm{L}$-drop of cold PBS. First, wings and legs were removed. Surgical forceps were used to gently separate the insect head from the body and exposing the foregut. The abdominal cuticle was then cut and dissected out, and the hindgut pulled outside the abdominal cavity. To completely free the entire gastrointestinal tract, the insect head and Malpighian tubes were removed. Only undamaged organs were further processed. Dissected guts, three per experimental condition, were immediately transferred into vials containing $100 \mu \mathrm{L}$ of cold PBS and kept at $-80{ }^{\circ} \mathrm{C}$ until use. A total of 15 nej and 15 yw flies were processed.

Bacterial DNA was extracted by means of QIAamp DNA Microbiome Kit (Qiagen, Hilden, Germany), designed to achieve enrichment of bacterial DNA from low biomass samples. Briefly, the depletion of host cells was performed by adding lysis buffer and benzonase to samples. Bacterial cell lysis was carried out by bead beating in the TissueLyser LT instrument (Qiagen, Hilden, Germany). Lysates were transferred to QIAamp UCP Mini Columns and processed according to the manufacturer's instructions. DNA was eluted in $30 \mu \mathrm{L}$ of the provided buffer. Library preparation and sequencing were performed as described above for human samples on the Illumina platform.

\subsection{Statistical Analysis}

Biological cell data were analyzed using Prism software (GraphPad Software, Sand Diego, CA, USA) and expressed as mean \pm Standard Deviation (SD). Student's t-tests were used to compare means between groups in AlphaLISA, Ki67, and TUNEL assays (LCLs acetylation, proliferation, and death rate), and in phenotypic evaluation (Drosophila embryo survival), with $p<0.05$ considered significant ${ }^{*} p<0.05 ;{ }^{* *} p<0.01$; ${ }^{* *} p<0.001$ for graphics relative to in vitro model); the correlation between HDACi-induced acetylation and proliferative or apoptotic cells was calculated using Pearson correlation coefficient $(-1<\mathrm{r}<1)$ and Pearson correlation $p$-value, significant for $p<0.05$.

For microbiota analysis, statistical evaluation among alpha-diversity indices was performed by a non-parametric Monte Carlo-based test, using 9999 random permutations. The PERMANOVA test (adonis function) in the $R$ package vegan (version 2.0-10) was used to compare the microbial community structure of RSTS and HD subjects within the beta-diversity analysis. For evaluating differences in taxonomic relative abundances, the pairwise t-test from the package "rstatix" (version 0.6.0) in the RStudio software (version 1.2.1335; $\mathrm{R}$ version 3.6.3) was used. $p$-values $<0.05$ were considered significant for each analysis.

Supplementary Materials: The following are available online at https:/ / www.mdpi.com/article / 10.3390/ijms22073621/s1, Figure S1: Insight on single-RSTS LCLs histone acetylation, Figure S2: HDAC inhibitors cytotoxicity analysis on RSTS LCLs, Figure S3: Insights on cell proliferation and cell death rate of RSTS LCLs upon HDAC inhibitors exposure, Figure S4: Correlation between HDACi-induced acetylation versus cell proliferation and apoptosis in RSTS LCLs, Figure S5: Gut microbiota composition in HD and RSTS subjects, Figure S6: Normal and altered phenotypes of nej mutant embryos from stage 8 to 12 treated or not with HDACi, Table S1: RSTS LCLs used for in vitro treatments, Table S2: Conditions of in vitro treatments used on LCLs, Table S3: Nutritional values of the enrolled patients, Table S4: Gut microbiota composition in HD and RSTS subjects.

Author Contributions: V.M., E.B. and C.G. conceived the project; E.D.F., E.O., P.G., A.G., C.P. and G.F. performed the experiments; C.C. and M.S. performed bioinformatics analyses; E.A.C. and G.B. contributed to molecular analyses; D.M. contributed to patient recruitment and clinical assessment; T.V. provided flies, reagents, animals, and design of in vivo experiments; E.D.F., E.O., V.M., E.B. and C.G. performed data analysis and interpretation; E.D.F., E.O., V.M., E.B. and C.G. wrote the manuscript; E.V., D.M. and T.V. provided guidance in the manuscript revision. All authors have read and agreed to the published version of the manuscript. 
Funding: This work was supported by Fondazione Cariplo (2015-0783 to V.M.), by intramural funding (Università degli Studi di Milano linea 2 to C.G.), by Associazione "RTS Una Vita Speciale ONLUS" (\#DigiRare to C.G.), by grant "Aldo Ravelli Center" (to V.M. and C.G.); by Translational Medicine PhD-Università degli Studi di Milano scholarship (to E.D.F. and C.P.); by Molecular and Translational Medicine PhD-Università degli Studi di Milano scholarship (to E.O., C.C. and P.G.), by H2020 Marie Skłodowska-Curie Actions (H2020-MSCA individual fellowship \#844147) (to A.G.).

Institutional Review Board Statement: The study was conducted according to the guidelines of the Declaration of Helsinki and its later amendments. The use of lymphoblastoid cell lines was approved by Ethics Committee of Università degli Studi di Milano (Comitato Etico number 99/20). The study on gut microbiota profiling of the enrolled subjects was approved by Ethics Committee of San Paolo Hospital in Milan (Comitato Etico Milano Area 1, Protocol number 2019/EM/076).

Informed Consent Statement: Informed consent was obtained from all subjects involved in the study or their caregivers.

Data Availability Statement: Sequencing data of 16S rRNA amplicons have been deposited in NCBI Short-Read Archive (SRA) under accession number PRJNA616211 (http:/ / www.ncbi.nlm.nih.gov / bioproject/PRJNA616211, accessed on 22 February 2021).

Acknowledgments: We thank the patients' families for participating in this study. C.G. thanks the Italian Association of Rubinstein-Taybi patients "RTS Una Vita Speciale ONLUS" for its support. This work belongs to the ERN ITHACA network (DM). Microscopy observations were carried out at The Advanced Microscopy Facility Platform-UNItech NOLIMITS-University of Milan. All authors acknowledge support from the University of Milan through the APC initiative.

Conflicts of Interest: The authors declare no conflict of interest.

\section{References}

1. Atlasi, Y.; Stunnenberg, H.G. The interplay of epigenetic marks during stem cell differentiation and development. Nat. Rev. Genet. 2017, 18, 643-658. [CrossRef] [PubMed]

2. Xu, Q.; Xie, W. Epigenome in Early Mammalian Development: Inheritance, Reprogramming and Establishment. Trends Cell Biol. 2018, 28, 237-253. [CrossRef] [PubMed]

3. Grunstein, M. Histone acetylation in chromatin structure and transcription. Nature 1997, 389, 349-352. [CrossRef]

4. Uchida, S.; Shumyatsky, G.P. Epigenetic regulation of Fgf1 transcription by CRTC1 and memory enhancement. Brain Res. Bull. 2018, 141, 3-12. [CrossRef]

5. Bjornsson, H.T. The Mendelian disorders of the epigenetic machinery. Genome Res. 2015, 25, 1473-1481. [CrossRef] [PubMed]

6. Rubinstein, J.H.; Taybi, H. Broad thumbs and toes and facial abnormalities. A possible mental retardation syndrome. Am. J. Dis. Child. 1963, 105, 588-608. [CrossRef] [PubMed]

7. Hennekam, R.C.M. Rubinstein-Taybi syndrome. Eur. J. Hum. Genet. 2006, 14, 981-985. [CrossRef]

8. Milani, D.; Manzoni, F.; Pezzani, L.; Ajmone, P.; Gervasini, C.; Menni, F.; Esposito, S. Rubinstein-Taybi syndrome: Clinical features, genetic basis, diagnosis, and management. Ital. J. Pediatr. 2015, 41, 4. [CrossRef]

9. Fergelot, P.; Van Belzen, M.; Van Gils, J.; Afenjar, A.; Armour, C.M.; Arveiler, B.; Beets, L.; Burglen, L.; Busa, T.; Collet, M.; et al. Phenotype and genotype in 52 patients with Rubinstein-Taybi syndrome caused by EP300 mutations. Am. J. Med. Genet. Part A 2016, 170, 3069-3082. [CrossRef]

10. Miller, R.W.; Rubinstein, J.H. Tumors in Rubinstein-Taybi syndrome. Am. J. Med. Genet. 1995, 56, 112-115. [CrossRef]

11. Boot, M.V.; van Belzen, M.J.; Overbeek, L.I.; Hijmering, N.; Mendeville, M.; Waisfisz, Q.; Wesseling, P.; Hennekam, R.C.; de Jong, D. Benign and malignant tumors in Rubinstein-Taybi syndrome. Am. J. Med. Genet. Part A 2018, 176, 597-608. [CrossRef]

12. Valor, L.M.; Viosca, J.; Lopez-Atalaya, J.P.; Barco, A. Lysine acetyltransferases CBP and p300 as therapeutic targets in cognitive and neurodegenerative disorders. Curr. Pharm. Des. 2013, 19, 5051-5064. [CrossRef] [PubMed]

13. Yao, T.P.; Oh, S.P.; Fuchs, M.; Zhou, N.D.; Ch'ng, L.E.; Newsome, D.; Bronson, R.T.; Li, E.; Livingston, D.M.; Eckner, R. Gene dosage-dependent embryonic development and proliferation defects in mice lacking the transcriptional integrator p300. Cell 1998, 93, 361-372. [CrossRef]

14. Oike, Y.; Hata, A.; Mamiya, T.; Kaname, T.; Noda, Y.; Suzuki, M.; Yasue, H.; Nabeshima, T.; Araki, K.; Yamamura, K. Truncated CBP protein leads to classical Rubinstein-Taybi syndrome phenotypes in mice: Implications for a dominant-negative mechanism. Hum. Mol. Genet. 1999, 8, 387-396. [CrossRef] [PubMed]

15. Chan, H.M.; La Thangue, N.B. p300/CBP proteins: HATs for transcriptional bridges and scaffolds. J. Cell Sci. 2001, 114, 2363-2373.

16. Dutto, I.; Scalera, C.; Prosperi, E. CREBBP and p300 lysine acetyl transferases in the DNA damage response. Cell. Mol. Life Sci. 2018, 75, 1325-1338. [CrossRef] [PubMed] 
17. Weinert, B.T.; Narita, T.; Satpathy, S.; Srinivasan, B.; Hansen, B.K.; Schölz, C.; Hamilton, W.B.; Zucconi, B.E.; Wang, W.W.; Liu, W.R.; et al. Time-Resolved Analysis Reveals Rapid Dynamics and Broad Scope of the CBP/p300 Acetylome. Cell 2018, 174, 231-244.e12. [CrossRef]

18. Kazantsev, A.G.; Thompson, L.M. Therapeutic application of histone deacetylase inhibitors for central nervous system disorders. Nat. Rev. Drug Discov. 2008, 7, 854-868. [CrossRef] [PubMed]

19. Heerboth, S.; Lapinska, K.; Snyder, N.; Leary, M.; Rollinson, S.; Sarkar, S. Use of epigenetic drugs in disease: An overview. Genet. Epigenet. 2014, 6, 9-19. [CrossRef] [PubMed]

20. Lopez-Atalaya, J.P.; Gervasini, C.; Mottadelli, F.; Spena, S.; Piccione, M.; Scarano, G.; Selicorni, A.; Barco, A.; Larizza, L. Histone acetylation deficits in lymphoblastoid cell lines from patients with Rubinstein-Taybi syndrome. J. Med. Genet. 2012, 49, 66-74. [CrossRef]

21. Alarcón, J.M.; Malleret, G.; Touzani, K.; Vronskaya, S.; Ishii, S.; Kandel, E.R.; Barco, A. Chromatin Acetylation, Memory, and LTP Are Impaired in CBP+/ - Mice. Neuron 2004, 42, 947-959. [CrossRef]

22. Simon, G.M.; Cheng, J.; Gordon, J.I. Quantitative assessment of the impact of the gut microbiota on lysine epsilon-acetylation of host proteins using gnotobiotic mice. Proc. Natl. Acad. Sci. USA 2012, 109, 11133-11138. [CrossRef]

23. Stilling, R.M.; van de Wouw, M.; Clarke, G.; Stanton, C.; Dinan, T.G.; Cryan, J.F. The neuropharmacology of butyrate: The bread and butter of the microbiota-gut-brain axis? Neurochem. Int. 2016, 99, 110-132. [CrossRef] [PubMed]

24. Astbury, S.M.; Corfe, B.M. Uptake and metabolism of the short-chain fatty acid butyrate, a critical review of the literature. Curr. Drug Metab. 2012, 13, 815-821. [CrossRef] [PubMed]

25. Eckschlager, T.; Plch, J.; Stiborova, M.; Hrabeta, J. Histone deacetylase inhibitors as anticancer drugs. Int. J. Mol. Sci. 2017, 18 , 1414. [CrossRef] [PubMed]

26. Italian Society of Human Nutrition Nutrients and Energy Reference Intake Levels for Italian Population. Available online: https:/ / sinu.it/tabelle-larn-2014/ (accessed on 18 February 2021).

27. Akimaru, H.; Chen, Y.; Dai, P.; Hou, D.-X.; Nonaka, M.; Smolik, S.M.; Armstrong, S.; Goodman, R.H.; Ishii, S. Drosophila CBP is a co-activator of cubitus interruptus in hedgehog signalling. Nature 1997, 386, 735-738. [CrossRef] [PubMed]

28. Akimaru, H.; Hou, D.-X.; Ishii, S. Drosophila CBP is required for dorsal-dependent twist gene expression. Nat. Genet. 1997, 17, 211-214. [CrossRef] [PubMed]

29. Cobos, S.N.; Bennett, S.A.; Torrente, M.P. The impact of histone post-translational modifications in neurodegenerative diseases. Biochim. Biophys. Acta Mol. Basis Dis. 2019, 1865, 1982-1991. [CrossRef] [PubMed]

30. José-Enériz, E.S.; Gimenez-Camino, N.; Agirre, X.; Prosper, F. HDAC inhibitors in acute myeloid leukemia. Cancers 2019, 11, 1794. [CrossRef]

31. Spartalis, E.; Athanasiadis, D.I.; Chrysikos, D.; Spartalis, M.; Boutzios, G.; Schizas, D.; Garmpis, N.; Damaskos, C.; Paschou, S.A.; Ioannidis, A.; et al. Histone deacetylase inhibitors and anaplastic thyroid carcinoma. Anticancer Res. 2019, 39, 1119-1127. [CrossRef]

32. Tarnowski, M.; Tkacz, M.; Kopytko, P.; Bujak, J.; Piotrowska, K.; Pawlik, A. Trichostatin A Inhibits Rhabdomyosarcoma Proliferation and Induces Differentiation through MyomiR Reactivation. Folia Biol. 2019, 65, 43-52.

33. Lipska, K.; Gumieniczek, A.; Filip, A.A. Anticonvulsant valproic acid and other short-chain fatty acids as novel anticancer therapeutics: Possibilities and challenges. Acta Pharm. 2020, 70, 291-301. [CrossRef] [PubMed]

34. Yuan, X.G.; Huang, Y.R.; Yu, T.; Jiang, H.W.; Xu, Y.; Zhao, X.Y. Chidamide, a histone deacetylase inhibitor, induces growth arrest and apoptosis in multiple myeloma cells in a caspase-dependent manner. Oncol. Lett. 2019, 18, 411-419. [CrossRef] [PubMed]

35. Iannitti, T.; Palmieri, B. Clinical and experimental applications of sodium phenylbutyrate. Drugs $R$ D 2011, 11, 227-249. [CrossRef]

36. Cappellacci, L.; Perinelli, D.R.; Maggi, F.; Grifantini, M.; Petrelli, R. Recent Progress in Histone Deacetylase Inhibitors as Anticancer Agents. Curr. Med. Chem. 2018, 27, 2449-2493. [CrossRef] [PubMed]

37. Davie, J.R. Inhibition of Histone Deacetylase Activity by Butyrate. J. Nutr. 2003, 133, 2485S-2493S. [CrossRef]

38. Louis, P.; Flint, H.J. Diversity, metabolism and microbial ecology of butyrate-producing bacteria from the human large intestine. FEMS Microbiol. Lett. 2009, 294, 1-8. [CrossRef]

39. Borghi, E.; Vignoli, A. Rett syndrome and other neurodevelopmental disorders share common changes in gut microbial community: A descriptive review. Int. J. Mol. Sci. 2019, 20, 4160. [CrossRef] [PubMed]

40. Lindefeldt, M.; Eng, A.; Darban, H.; Bjerkner, A.; Zetterström, C.K.; Allander, T.; Andersson, B.; Borenstein, E.; Dahlin, M.; Prast-Nielsen, S. The ketogenic diet influences taxonomic and functional composition of the gut microbiota in children with severe epilepsy. Npj Biofilms Microbiomes 2019, 5, 5. [CrossRef] [PubMed]

41. Benjamin, J.S.; Pilarowski, G.O.; Carosso, G.A.; Zhang, L.; Huso, D.L.; Goff, L.A.; Vernon, H.J.; Hansen, K.D.; Bjornsson, H.T. A ketogenic diet rescues hippocampal memory defects in a mouse model of Kabuki syndrome. Proc. Natl. Acad. Sci. USA 2017, 114, 125-130. [CrossRef] [PubMed]

42. Heijtz, R.D.; Wang, S.; Anuar, F.; Qian, Y.; Björkholm, B.; Samuelsson, A.; Hibberd, M.L.; Forssberg, H.; Pettersson, S. Normal gut microbiota modulates brain development and behavior. Proc. Natl. Acad. Sci. USA 2011, 108, 3047-3052. [CrossRef] [PubMed]

43. Bach Knudsen, K.E.; Lærke, H.N.; Hedemann, M.S.; Nielsen, T.S.; Ingerslev, A.K.; Gundelund Nielsen, D.S.; Theil, P.K.; Purup, S.; Hald, S.; Schioldan, A.G.; et al. Impact of Diet-Modulated Butyrate Production on Intestinal Barrier Function and Inflammation. Nutrients 2018, 10, 1499. [CrossRef] 
44. Tanaka, Y.; Naruse, I.; Maekawa, T.; Masuya, H.; Shiroishi, T.; Ishii, S. Abnormal skeletal patterning in embryos lacking a single Cbp allele: A partial similarity with Rubinstein-Taybi syndrome. Proc. Natl. Acad. Sci. USA 1997, 94, 10215-10220. [CrossRef] [PubMed]

45. Matzkin, L.M.; Johnson, S.; Paight, C.; Markow, T.A. Preadult Parental Diet Affects Offspring Development and Metabolism in Drosophila melanogaster. PLoS ONE 2013, 8, e59530. [CrossRef]

46. Capo, F.; Wilson, A.; Di Cara, F. The intestine of Drosophila melanogaster: An emerging versatile model system to study intestinal epithelial homeostasis and host-microbial interactions in humans. Microorganisms 2019, 7, 336. [CrossRef] [PubMed]

47. Fast, D.; Duggal, A.; Foley, E. Monoassociation with Lactobacillus plantarum Disrupts Intestinal Homeostasis in Adult Drosophila melanogaster. mBio 2018, 9, e01114-e01118. [CrossRef]

48. Shin, S.C.; Kim, S.-H.; You, H.; Kim, B.; Kim, A.C.; Lee, K.-A.; Yoon, J.-H.; Ryu, J.-H.; Lee, W.-J. Drosophila microbiome modulates host developmental and metabolic homeostasis via insulin signaling. Science 2011, 334, 670-674. [CrossRef]

49. Sharon, G.; Segal, D.; Ringo, J.M.; Hefetz, A.; Zilber-Rosenberg, I.; Rosenberg, E. Commensal bacteria play a role in mating preference of Drosophila melanogaster. Proc. Natl. Acad. Sci. USA 2010, 107, 20051-20056. [CrossRef] [PubMed]

50. Babu, A.; Kamaraj, M.; Basu, M.; Mukherjee, D.; Kapoor, S.; Ranjan, S.; Swamy, M.M.; Kaypee, S.; Scaria, V.; Kundu, T.K.; et al. Chemical and genetic rescue of an ep300 knockdown model for Rubinstein Taybi Syndrome in zebrafish. Biochim. Biophys. Acta Mol. Basis Dis. 2018, 1864, 1203-1215. [CrossRef]

51. Spena, S.; Milani, D.; Rusconi, D.; Negri, G.; Colapietro, P.; Elcioglu, N.; Bedeschi, F.; Pilotta, A.; Spaccini, L.; Ficcadenti, A.; et al. Insights into genotype-phenotype correlations from CREBBP point mutation screening in a cohort of 46 Rubinstein-Taybi syndrome patients. Clin. Genet. 2015, 88, 431-440. [CrossRef]

52. Negri, G.; Milani, D.; Colapietro, P.; Forzano, F.; Della Monica, M.; Rusconi, D.; Consonni, L.; Caffi, L.G.; Finelli, P.; Scarano, G.; et al. Clinical and molecular characterization of Rubinstein-Taybi syndrome patients carrying distinct novel mutations of the EP300 gene. Clin. Genet. 2015, 87, 148-154. [CrossRef] [PubMed]

53. Negri, G.; Magini, P.; Milani, D.; Colapietro, P.; Rusconi, D.; Scarano, E.; Bonati, M.T.; Priolo, M.; Crippa, M.; Mazzanti, L.; et al. From Whole Gene Deletion to Point Mutations of EP300-Positive Rubinstein-Taybi Patients: New Insights into the Mutational Spectrum and Peculiar Clinical Hallmarks. Hum. Mutat. 2016, 37, 175-183. [CrossRef] [PubMed]

54. Schölz, C.; Weinert, B.T.; Wagner, S.A.; Beli, P.; Miyake, Y.; Qi, J.; Jensen, L.J.; Streicher, W.; McCarthy, A.R.; Westwood, N.J.; et al. Acetylation site specificities of lysine deacetylase inhibitors in human cells. Nat. Biotechnol. 2015, 33, 415-423. [CrossRef]

55. Chang, M.C.; Chen, Y.J.; Lian, Y.C.; Chang, B.E.; Huang, C.C.; Huang, W.L.; Pan, Y.H.; Jeng, J.H. Butyrate stimulates histone H3 acetylation, 8-isoprostane production, RANKL expression, and regulated osteoprotegerin expression/secretion in MG-63 osteoblastic cells. Int. J. Mol. Sci. 2018, 19, 4071. [CrossRef] [PubMed]

56. Freese, K.; Seitz, T.; Dietrich, P.; Lee, S.M.L.; Thasler, W.E.; Bosserhoff, A.; Hellerbrand, C. Histone deacetylase expressions in hepatocellular carcinoma and functional effects of histone deacetylase inhibitors on liver cancer cells in vitro. Cancers 2019, 11, 1587. [CrossRef]

57. Tarasenko, N.; Chekroun-Setti, H.; Nudelman, A.; Rephaeli, A. Comparison of the anticancer properties of a novel valproic acid prodrug to leading histone deacetylase inhibitors. J. Cell. Biochem. 2018, 119, 3417-3428. [CrossRef]

58. Gottlicher, M.; Minucci, S.; Zhu, P.; Krämer, O.H.; Schimpf, A.; Giavara, S.; Sleeman, J.P.; Lo Coco, F.; Nervi, C.; Pelicci, P.G.; et al. Valproic acid defines a novel class of HDAC inhibitors inducing differentiation of transformed cells. PubMed NCBI EMBO J. 2001, 20, 6969-6978. [CrossRef]

59. Chriett, S.; Dąbek, A.; Wojtala, M.; Vidal, H.; Balcerczyk, A.; Pirola, L. Prominent action of butyrate over $\beta$-hydroxybutyrate as histone deacetylase inhibitor, transcriptional modulator and anti-inflammatory molecule. Sci. Rep. 2019, 9, 742. [CrossRef] [PubMed]

60. Verduci, E.; Moretti, F.; Bassanini, G.; Banderali, G.; Rovelli, V.; Casiraghi, M.C.; Morace, G.; Borgo, F.; Borghi, E. Phenylketonuric diet negatively impacts on butyrate production. Nutr. Metab. Cardiovasc. Dis. 2018, 28, 385-392. [CrossRef] [PubMed]

61. Masella, A.P.; Bartram, A.K.; Truszkowski, J.M.; Brown, D.G.; Neufeld, J.D. PANDAseq: Paired-end assembler for illumina sequences. BMC Bioinf. 2012, 13, 31. [CrossRef] [PubMed]

62. Caporaso, J.G.; Kuczynski, J.; Stombaugh, J.; Bittinger, K.; Bushman, F.D.; Costello, E.K.; Fierer, N.; Pẽa, A.G.; Goodrich, J.K.; Gordon, J.I.; et al. QIIME allows analysis of high-throughput community sequencing data. Nat. Methods 2010, 7, 335-336. [CrossRef] [PubMed]

63. Wang, Q.; Garrity, G.M.; Tiedje, J.M.; Cole, J.R. Naïve Bayesian classifier for rapid assignment of rRNA sequences into the new bacterial taxonomy. Appl. Environ. Microbiol. 2007, 73, 5261-5267. [CrossRef]

64. Lozupone, C.; Lladser, M.E.; Knights, D.; Stombaugh, J.; Knight, R. UniFrac: An effective distance metric for microbial community comparison. ISME J. 2011, 5, 169-172. [CrossRef] [PubMed]

65. Bassanini, G.; Ceccarani, C.; Borgo, F.; Severgnini, M.; Rovelli, V.; Morace, G.; Verduci, E.; Borghi, E. Phenylketonuria Diet Promotes Shifts in Firmicutes Populations. Front. Cell. Infect. Microbiol. 2019, 9, 101. [CrossRef] [PubMed]

66. Ashburner, M. Drosophila: A Laboratory Handbook; Cold Spring Harbor Laboratory Press: New York, NY, USA, 1989. 